\title{
Harmonic Mappings and Disc Bundles over Compact Kähler Manifolds
}

By

\author{
Klas DiEDERICH* and Takeo OHSAWA**
}

\section{Introduction and Results}

Let $M$ be a complex manifold and $\Omega \Subset M$ a domain. For the convenience of the reader and in order to avoid any confusion we repeat the following definitions.

Definition. a) $\Omega$ is called locally pseudoconvex near $z_{0} \in \partial \Omega$ if there exists a neighborhood $U=U\left(z_{0}\right)$ and a plurisubharmonic $C^{\infty}$ function $\varphi$ on $U \cap \Omega$ such that

$$
\{\overline{z \in U \cap \Omega: \varphi(z)<c\}} \cap \partial \Omega=\phi \quad \forall c \in \mathbb{R} .
$$

Such a function $\varphi$ is called a local plurisubharmonic exhaustion function of $\Omega$ near $z_{0}$, and $\Omega$ is called pseudoconvex, if it is locally pseudoconvex near all $z_{0} \in \partial \Omega$.

b) $\Omega$ is called weakly 1 -complete if there is a $C^{\infty}$ plurisubharmonic function $\varphi$ on $\Omega$ with

$$
\{z \in \Omega: \varphi(z)<c\} \Subset \Omega \quad \forall c \in \mathbb{R}
$$

(a $C^{\infty}$ plurisubharmonic exhaustion function).

The following result is well-known:

Theorem. If $M$ is a Stein manifold and $\Omega \Subset M$ a domain, then the following conditions on $\Omega$ are equivalent:

i) $\Omega$ is pseudoconvex,

ii) $\Omega$ is weakly 1-complete,

* Mathematik, Bergische Universität-GHS, Gaußstraße 20, Wuppertal 1, West Germany.

** Research Institute for Mathematical Sciences, Kyoto University, Kyoto 606, Japan. 

iii) $\Omega$ is 1-convex,
iv) $\Omega$ is holomorphically convex,
v) $\Omega$ is Stein.

For an arbitrary complex manifold $M$ and a domain $\Omega \Subset M$ one still has (with the same notation as in the previous theorem):

Theorem. v) $\Rightarrow \mathrm{iv}) \Rightarrow$ iii) $\Rightarrow \mathrm{ii}) \Rightarrow \mathrm{i}$ ) and also iii) $\Rightarrow \mathrm{iv}$ ).

The deep fact that iii) $\Rightarrow$ iv) was first shown by Grauert in [5]. On the other hand, it is also known from concrete counterexamples by H. Grauert [8], [6] and others that in general:

ii) $\nrightarrow$ iii) and iv) $\nRightarrow$ v).

Furthermore, in [3] Diederich and Fornaess constructed a locally trivial holomorphic disc bundle $\Omega \rightarrow X$ over the Hopf surface $X=\mathbb{C}^{2}$ / $(z \sim 2 z)$ which is, of course, pseudoconvex in the corresponding $\mathbb{P}^{1}$ bundle $\hat{\Omega} \rightarrow X$, but, nevertheless, cannot be exhausted by relatively compact pseudoconvex domains. In particular, $\Omega$ is not weakly $1-$ complete, such that, in general

$$
\text { i) } A \text { ii). }
$$

In other words, even for plurisubharmonic exhaustion functions the passage from the local to the global situation is non-trivial (as in the case of the Levi-problem). Since, however, weakly 1-complete manifolds satisfy several vanishing and finiteness theorems (for a survey of these results see T. Ohsawa [9]), one would like to know under which additional assumptions pseudoconvex domains are necessarily weakly 1-complete. This article is a contribution towards this question. We want to show:

Theorem 1. Let $X$ be a compact Kähler manifold and $\Omega^{\pi} \rightarrow X$ a locally trivial holomorphic disc bundle. Then $\Omega$ is weakly 1-complete.

Remark. Theorem 1 will be generalized in $\S 5$, which also contains an extendibility result for harmonic maps.

The proof is another example for the usefulness of harmonic maps in complex analysis, the idea being as follows: 
Suppose, there exists a holomorphic section $s: X \rightarrow \Omega$, i。 e. $\pi \circ s=\mathrm{id}$. Then the function

$$
\varphi(p):=\operatorname{dist}^{2}(p, h \circ \pi(p))
$$

where dist $(\cdot, \cdot)$ is the Poincaré distance in the fiber $\pi^{-1} \circ \pi(p)$, is a plurisubharmonic $C^{\infty}$ exhaustion of $\Omega$, as can easily be seen. However, most bundles $\Omega \rightarrow X$ do not allow holomorphic sections. Therefore, we try to replace them by harmonic sections, where "harmonic" refers to a given Kähler metric $d s_{X}^{2}$ on $X$ and the Kähler metric $d s^{2}$ induced on $\Omega$ by $d s_{X}^{2}$ and the Poincaré metric $d s_{h}^{2}$ on $\Delta$. (Notice that because of the hyperbolicity of $\Delta$ the transition functions of $\Omega^{\pi} \rightarrow X$

$$
\phi_{i j}: U_{i} \cap U_{j} \rightarrow \text { Aut } \Delta
$$

for a trivializing open covering $\left\{U_{i}\right\}$ of $X$ are locally constant.) For definitions and elementary facts about harmonic mappings we refer the reader to $\S 1$ and Eells-Sampson [4], Hamilton [7] and Siu [13]. The crucial part of the proof of Theorem 1 is then the following existence theorem for harmonic sections.

Theorem 2. Let $\Omega \stackrel{\pi}{\rightarrow} X$ be as in Theorem 1 and suppose that the corresponding $\mathbb{P}^{1}$-bundle $\hat{\Omega}^{*} \rightarrow X$ does not allow a flat section in $\partial \Omega$. Then there exists a harmonic section $s: X \rightarrow \Omega$.

Remark. A section $s: X \rightarrow \widehat{\Omega}$ is called flat if its fiber coordinates over any connected trivializing open set $U \subset X$ are constant.

In the situation of Theorem 2 a function $\varphi$ similar to (1) will do the job, as we will show in $\S 2$ by using an important result of Siu [13]. For bundles where $\hat{\Omega} \rightarrow X$ has a flat section in $\partial \Omega$ Theorem 1 has to be proved by a different method (see $\S 4$ ).

For the proof of Theorem 2 we use the method of Eells-Sampson [4] (see also Hamilton [7]). More precisely, we want to deform an arbitrary given $C^{\infty}$ section $s_{0}: X \rightarrow \Omega$ into a harmonic section $s: X \rightarrow \Omega$ by taking $s_{0}$ as initial condition tor the heat equation

$$
\frac{\partial}{\partial t} s(p, t)=A_{p} s(p, t)
$$

associated with the generalized Laplace operator $A$. Two difficulties arise :

1) The curvature of $d s^{2}$ need not be negative as is required in 
[4] and [7] and other existence theorems for harmonic maps, 2) $\Omega$ is not compact.

The first problem can be overcome because $s_{0}$ is a section and, at least, the curvature of $d s_{h}^{2}$ is negative.

The second problem is solved by showing that a solution $s(p, t)$ of (2), roughly speaking, converges for increasing $t$ either to a harmonic section of $\Omega^{\pi} \rightarrow X$ or to a flat section of $\hat{\Omega}^{*} \rightarrow X$. For details see $\S 3$.

The first author learned a lot about harmonic maps during a visit to the Institute for Advanced Study, Princeton in the academic year 1982/83 in conversations with J. Eells whom he would like to thank for his patience in answering many questions. And he also wants to thank the Institute for its hospitality and support.

\section{$\S 1$. Harmonic and Pluriharmonic Maps}

For the convenience of the reader we will put together in this section some basic definitions and formulas concerning harmonic and pluriharmonic maps.

We always suppose that $M$ and $N$ are Kähler manifolds with fixed Kähler metrics $d s_{M}^{2}$ and $d s_{N}^{2}$, the metric tensors of which are denoted by $g_{M}$ and $g_{N}$. Furthermore,

$$
f: M \rightarrow N
$$

is a fixed $C^{\infty}$ map.

a) The manifolds $\left(M, d s_{M}^{2}\right)$ and $\left(N, d s_{N}^{2}\right)$ can be considered as Riemannian manifolds in the usual way. Then the fundamental form $\beta(f)$ of $f$ is defined as $\beta(f)=\nabla d f$ where $\nabla$ is covariant differentiation with respect to the connection induced on $T^{*} M \otimes f^{*} T N$. The map $f$ is totally geodesic if

$$
\beta(f) \equiv 0
$$

and if is called harmonic if

$$
A f:=\operatorname{trace} \beta(f) \equiv 0 .
$$

b) Since $M$ and $N$ are hermitian manifolds, one also can consider the tensor $\nabla_{1,0} \bar{\partial} f$, which is a section of $T^{* 1,0} M \otimes T^{* 0,1} M \otimes f^{*} T^{1,0} N$, and represents one part of $\beta(f)$. In local coordinates one has

$$
\left(\nabla_{1,0} \bar{\partial} f\right)_{i j}^{\alpha}=f_{i j}^{\alpha}+\Gamma_{N, \beta r}^{\alpha}(f) f_{i}^{\beta} f_{j}^{r}-\Gamma_{M, i j}^{k} f_{k}^{\alpha} .
$$

Notice that on Kähler manifolds $M$ one has $\Gamma_{M, i j}^{k} \equiv 0$. 
Definition 1. The map $f$ is called pluriharmonic if $\nabla_{1,0} \bar{\partial} f \equiv 0$ 。

Remark. For $M$ Kähler, pluriharmonicity obviously does not depend on the choice of $d s_{M}^{2}$.

Simple calculations show that for $M$ Kähler the following formula holds

$$
(\Delta f)^{\alpha}=2 g^{i j}\left\{f_{i j}^{\alpha}+\Gamma_{N, \beta r}^{\alpha}(f) f_{i}^{\beta} f_{j}^{r}\right\} .
$$

In particular, pluriharmonic maps are indeed harmonic.

We will need in $\S 3$ the following two notions of energy which play a fundamental role in the theory of harmonic maps:

Definition 2. a) For an arbitrary $C^{\infty}$ map $f: M \rightarrow N$ we define $\chi:=\frac{1}{2}|\nabla f|^{2}$, where the length of $\nabla f$ is measured with respect to $d s_{M}^{2}$ and $d s_{N}^{2}$, as the potential energy density of $f$ on $M$.

b) Let $f: M \times[a, b] \rightarrow N$ be a $C^{\infty}$-mapping. Then the kinetic energy density of $\hat{f}$ is defined as $\kappa=\frac{1}{2}\left|\frac{\partial f}{\partial t}\right|^{2}$ where the length is measured with respect to $d s_{N}^{2}$.

\section{§2. Disc Bundles with Harmonic Sections}

In this section we want to show the following part of Theorem 1:

Proposition 1. Let $\Omega \stackrel{\pi}{\rightarrow} X$ be a locally trivial holomorphic disc bundle over a compact Kähler manifold $X$. Let $\Omega$ be equipped with the Kähler metric $d s^{2}$ induced by a Kähler metric $d s_{X}^{2}$ on $X$ and the Poincaré-metric $d s_{\Delta}^{2}$ on 4 . Suppose there exists a harmonic section $s:\left(X, d s_{X}^{2}\right) \rightarrow\left(\Omega, d s^{2}\right)$ (in the sense of (1)). Then $\Omega$ is weakly 1-complete.

The proof of Prop. 1 is based on the following

Key lemma. Let $\left(\Delta, d s_{\Delta}^{2}\right)$ be the unit disc with the Poincare metric and $h:\left(\Delta, d s_{\Delta}^{2}\right) \rightarrow\left(\Delta, d s_{\Delta}^{2}\right)$ a harmonic map. Define on the bidisc $\Delta^{2} \subset \mathbb{C}^{2}$ the function 


$$
\psi(\zeta, w):=-\log \left(1-\left|\frac{w-\zeta}{\bar{w} \zeta-1}\right|^{2}\right)
$$

Then the function $\varphi(z, w):=\phi(h(z), w)$ is plurisubharmonic on $\Delta^{2}$.

Proof. A simple calculation using (1) and (4), which can be left to the reader, shows that the harmonicity of $h$ is equivalent to the following differential equation

$$
\frac{\partial^{2} h}{\partial z \partial \bar{z}}+\frac{2 \bar{h}}{1-|h|^{2}} \frac{\partial h}{\partial z} \frac{\partial h}{\partial \bar{z}}=0 .
$$

We want to check that the Levi form of $\varphi$ is positive semidefinite at an arbitrary fixed point $\left(z_{0}, w_{0}\right) \in \Delta^{2}$. We choose an automorphism $T$ of $\Delta$ with $T\left(h\left(z_{0}\right)\right)=0$ and introduce new complex coordinates $\left(z^{\prime}, w^{\prime}\right)$ on $\Delta^{2}$ by putting $z^{\prime}=z$ and $w^{\prime}=T(w)$. Then we have to show that the Levi-form of the function

$$
\varphi^{\prime}\left(z^{\prime}, w^{\prime}\right)=\varphi\left(z^{\prime}, T^{-1} w^{\prime}\right)=\phi\left(h\left(z^{\prime}\right), T^{-1} w^{\prime}\right)
$$

is positive semidefinite at $\left(z_{0}, T\left(w_{0}\right)\right)$.

But, notice that

$$
\varphi^{\prime}\left(z^{\prime}, w^{\prime}\right)=\phi\left(T \circ h\left(z^{\prime}\right), w^{\prime}\right) .
$$

Furthermore, since $T$ is an isometry with respect to $d s_{h}^{2}$, the map

$$
T \circ h:\left(\Delta, d s_{\Delta}^{2}\right) \rightarrow\left(\Delta, d s_{\Delta}^{2}\right)
$$

is also harmonic. Because of $T \circ h\left(z_{0}\right)=0$ this shows that returning to the old notation we only have to estimate the Levi form of $\varphi$ at points $\left(z_{0}, w\right)$ with $h\left(z_{0}\right)=0$. One has at such points

$$
\begin{aligned}
& \varphi_{z \bar{z}}=\left|h_{z}\right|^{2}+\left|h_{\bar{z}}\right|^{2}-2 \operatorname{Re}\left(\bar{w}_{0}^{2} h_{z} h_{\bar{z}}\right) \geqq 0 \\
& \varphi_{w \bar{w}}=\left(1-\left|w_{0}\right|^{2}\right)^{-2} \\
& \varphi_{z \bar{w}}=-h_{z} .
\end{aligned}
$$

It, therefore, suffices to show that

$$
l_{\varphi}:=\varphi_{z \bar{z}} \varphi_{w \bar{w}}-\left|\varphi_{z \bar{w}}\right|^{2} \geqq 0 .
$$

One obtains easily

(5a) $l_{\varphi}=\left(1-\left|w_{0}\right|^{2}\right)^{-2}\left\{\left|w_{0}\right|^{2}\left(2-\left|w_{0}\right|^{2}\right)\left|h_{z}\right|^{2}+\left|h_{\bar{z}}\right|^{2}-2 \operatorname{Re}\left(\bar{w}_{0}^{2} h_{z} h_{\bar{z}}\right)\right\}$.

But

$$
\left|w_{0}\right|^{4}-\left|w_{0}\right|^{2}\left(2-\left|w_{0}\right|^{2}\right)=2\left|w_{0}\right|^{2}\left(\left|w_{0}\right|^{2}-1\right)<0 .
$$

Hence $l_{\varphi} \geqq 0$. 
How does Proposition 1 follow from the key lemma? The decisive step is contained in the following

Lemma 1. The given harmonic section $s: X \rightarrow \Omega$ is pluriharmonic.

Proof. We adopt a method developed by Y. T. Siu [13] to our situation.

For this we call $g$ the fundamental tensor of the Kähler metric $d s^{2}$ on $\Omega$ and denote by $R_{\Omega}$ its curvature tensor. Siu proved the following identity, which does not use the harmonicity of $s$ :

$$
\partial \bar{\partial}<g, \bar{\partial} s \wedge \partial \bar{s}\rangle=<R_{\Omega}, \bar{\partial} s \wedge \partial \bar{\partial} \wedge \partial s \wedge \bar{\partial} \bar{s}>-<g, \nabla_{1,0} \bar{\partial} s \wedge \nabla_{0,1} \partial \bar{s}>
$$

where $\langle\cdot, \cdot\rangle$ denotes contraction.

Over a trivializing neighborhood $U \subset X$ with $\Omega \mid U=U \times \Delta=\{(z, t)$ : $z \in U, t \in \Delta\}$ the section $s$ is of the form

$$
s(z)=(z, h(z))
$$

with a map $h: U \rightarrow \Delta$. Therefore, on $\Omega \mid U$ the identity (6) becomes

$$
\partial \bar{\partial}<g_{\Delta}, \bar{\partial} h \wedge \partial \bar{h}>=<R, \bar{\partial} h \wedge \partial \bar{h} \bigwedge \partial h \wedge \bar{\partial} \bar{h}>-<g_{\Delta}, \nabla_{1,0} \bar{\partial} h \wedge \nabla_{0,1} \partial \bar{h}>
$$

where $g_{\Delta}$ is the metric tensor of $d s_{h}^{2}$ on $\Delta_{X}$ and $R$ the corresponding curvature tensor. Since $R<0$ we can now apply identity (4.3) from [13] on $\Omega \mid U$ and obtain

$$
<R, \bar{\partial} h \wedge \partial \bar{h} \bigwedge \partial h \wedge \bar{\partial} \bar{h}>\wedge \omega^{n-2}=\sigma \omega^{n}
$$

with a nonnegative function $\sigma \geqq 0$ on $U$.

Therefore, we even have

$$
<R_{\Omega}, \bar{\partial} s \wedge \partial \bar{s} \wedge \partial s \wedge \bar{\partial} \bar{s}>\wedge \omega^{n-2}=\sigma \omega^{n}
$$

with a nonnegative function $\sigma$ on $X$.

Finally, we use the following formula (4.1) of [13], which requires the harmonicity of $s$ :

$$
<g, \nabla_{1,0} \bar{\partial} s \wedge \nabla_{0,1} \partial \bar{s}>\wedge \omega^{n-2}=\chi \omega^{n}
$$

with a function $\chi \leqq 0$ on $X$.

After substituting (8) and (9) in (6) and integrating over the compact manifold $X$ we obtain immediately

$$
\sigma=\chi=0 \text { on } X \text {. }
$$

Now Siu also shows in [13], p. 82, that $s$ is pluriharmonic if and only if $\chi \equiv 0$ in (9). This proves the lemma. 
Proof of Proposition 1: Let $U \subset X$ be a trivializing neighborhood for $\Omega \rightarrow X$ and $h: U \rightarrow \Delta$ as above. Since on $\Omega \mid U=U \times \Delta$ we have $d s^{2}=d s_{X}^{2} \oplus d s_{\Delta}^{2}$ the pluriharmonicity of $s$ implies that also $h$ is pluriharmonic. If now $f: \Delta \rightarrow U$ is any holomorphic map, also $h \circ f: \Delta \rightarrow \Delta$ is (pluri-)harmonic. We define on $\Omega \mid U$ the function

$$
\varphi(z, t):=\phi(h(z), t)
$$

with $\phi$ as in the key lemma.

Then $\phi(h \circ f(\zeta), t)$ on $\Delta \times \Delta$ is plurisubharmonic, proving that $\varphi$ is plurisubharmonic on $\Omega \mid U$.

On the other hand, because $\phi(h(z), t)=\psi(T(h(z)), T(t))$ for $T \in$ Aut $\Delta$ the function $\varphi$ is well defined on $\Omega$ and obviously also exhaustive.

\section{§ 3. Existence of Harmonic Sections}

In this section we want to prove Theorem 2. As indicated in the introduction we will construct the desired harmonic section by deforming an arbitrary $C^{\infty}$ section $s_{0}: X \rightarrow \Omega$ into a harmonic one. For this purpose, we use the heat equation for the generalized Laplacian $\Delta$ :

$$
\frac{d}{d t} s(p, t)=\Delta s(p, t)
$$

for a map $s: X \times[0, \tau] \rightarrow \Omega \tau>0$, according to the original method of Eells and Sampson [4]. As initial conditions we take

$$
s(p, 0):=s_{0}(p) .
$$

Since we, finally, are looking for a section, which is harmonic, it is natural to add to (10) and (11) the side condition

$$
\pi \circ s(p, t)=p \quad \forall p .
$$

At first, this might look like an additional complication. But we can show at first:

Lemma 2. Suppose $s$ is a solution of (10) and (11). Then (12) is automatically satisfied.

Proof. The map $\pi$ is obviously totally geodesic. Therefore, it 
follows from (10) that also

$$
\frac{d}{d t} \pi \circ s(p, t)=\Delta_{X} \pi \circ s(p, t)
$$

(see [4], p. 131) where $\Delta_{X}$ is the generalized Laplacian for maps from $X$ to $X$. As initial condition we obtain from (11)

$$
\pi \circ s(p, 0)=p \quad \forall p .
$$

But, since $X$ is compact the solutions of

$$
\frac{d}{d t} \tilde{s}(p, t)=A_{X} \tilde{s}(p, t)
$$

are uniquely determined by the initial condition and since $\tilde{s}(p, t) \equiv p$ is also a solution of (14) with (13) we obtain $\pi \circ s=i d: X \rightarrow X$.

As a next step we want to use the fact that solutions of (10) always exist for small periods of time (without any curvature assumption) :

Lemma 3. Let for some $t_{0} \geqq 0$ the $C^{\infty}$ map

$$
s\left(\bullet, t_{0}\right): X \rightarrow \Omega
$$

be given. Then there is an $\varepsilon>0$ and a uniquely determined $C^{\infty}$ map

$$
s: X \times\left[t_{0}, t_{0}+\varepsilon\right] \rightarrow \Omega
$$

such $s\left(\cdot, t_{0}\right)$ is the given map and $(10)$ is satisfied.

This is the theorem on p. 122 of [7] for $X$ compact.

Now we define

$$
\mu:=\sup \{\varepsilon: \exists s: X \times[0, \varepsilon] \rightarrow \Omega \text { with }(10) \text { and }(11)\} \leqq \infty .
$$

Because of the uniqueness in Lemma 3 we then have a $C^{\infty}$-map

$$
s: X \times[0, \mu) \rightarrow \Omega
$$

satisfying (10) and (11).

The following proposition contains the main result of the theory of Eells and Sampson (for simplicity we use R. S. Hamilton [7] as a reference). 
Proposition 2. Suppose that for any family s solving (10) the following two a priori-estimates for the potential energy density $\lambda$ and the kinetic energy density $\kappa$ of $s$ hold:

$$
\begin{aligned}
& \frac{\partial \chi}{\partial t} \leqq \Delta \chi+C \chi-|\nabla \nabla f|^{2} \\
& \frac{\partial \kappa}{\partial t} \leqq \Delta \kappa-\left|\nabla \frac{\partial f}{\partial t}\right|^{2} .
\end{aligned}
$$

Then one has:

a) The family $\left\{s_{t}=s(\cdot, t): t \in[0, \mu)\right\}$ is uniformly equicontinuous on $X$ with respect to $d s_{X}^{2}$ and $d s^{2}$.

b) If there is a compact set $K \subset \Omega$ such that $s_{t}(X) \subset K \quad \forall t \in[0, \mu)$ then $\mu=\infty$ and there exists a sequence $t_{1}<t_{2}<t_{3}<\cdots$ with $\lim _{k \rightarrow \infty} t_{k}=\infty$ such that $s_{\infty}:=\lim _{k \rightarrow \infty} s_{k}: X \rightarrow \Omega$ exists as a $C^{\infty}-m a p$. Any such $s_{\infty}$ is harmonic.

Proof. Part a) follows essentially from the corollary on p. 156, [7], if one only observes that the curvature conditions are only used in the proofs of the corollaries on pages 128 and 134, [7] (corresponding to the verifications of (15) and (16)). Part b) is the theorem in [7], p.157, the compactness assumption for the target being replaced by the assumption on $K$.

Next we have to ask why the à-priori estimates (15) and (16) are satisfied in our case.

Lemma 4. The estimates (15) and (16) hold for any family s solving (10).

Proof. Notice that the energy densities for $s$ split over any trivializing neighborhood $U \subset X$ with $s(z)=(z, h(z))$ as follows:

$$
\chi=\chi_{h}+n, \kappa=\kappa_{h}
$$

where $\chi_{h}\left(\right.$ resp. $\left.\kappa_{h}\right)$ is the potential (resp. kinetic) energy density of $h: U \rightarrow \Delta$. Since the curvature $R_{\Delta}$ is negative we obtain as on p. 128 resp. 134, [7], the following estimates on $U$ :

$$
\begin{aligned}
& \frac{\partial \chi_{h}}{\partial t} \leqq \Delta \chi_{h}+C \chi_{h}-|\nabla \nabla h|^{2} \\
& \frac{\partial \kappa_{h}}{\partial t} \leqq \Delta \kappa_{h}-\left|\nabla \frac{\partial \kappa_{h}}{\partial t}\right|^{2} \cdot
\end{aligned}
$$


From this (15) and (16) follow with (17).

Now we can easily give the

Proof of Proposition 1. We know from Lemma 4 that a) and b) of Prop. 2 hold. Suppose the assumption of b) is not satisfied. Since $X$ is compact, there is a point $p \in X$ and a sequence $t_{1}<t_{2}<t_{3}<\cdots$, $\lim t_{k}=\mu$ such that

$$
\exists \lim _{k \rightarrow \infty} s\left(p, t_{k}\right)=q \in \partial \Omega .
$$

Notice that on the other hand because of the uniform equicontinuity of $s_{t}$ there is a $C>0$ such that

$$
\operatorname{diam} s\left(X, t_{k}\right) \leqq C \quad \forall k
$$

in the metric $d s^{2}$. Because of the completeness of $d s^{2}$ we get immediately that

$$
s\left(X, t_{k}\right) \rightarrow \partial \Omega .
$$

But even more : since

$$
d s_{\Delta}^{2}(\zeta)=\frac{|d \zeta|^{2}}{\left(1-|\zeta|^{2}\right)^{2}}
$$

one obtains over each trivializing neighborhood $U \subset X$ with $s(z, t)=$ $(z, h(z, t))$

$$
\exists \lim _{k \rightarrow \infty} h\left(z, t_{k}\right)=\text { const. }
$$

Therefore, $\lim _{k \rightarrow \infty} s\left(\bullet, t_{k}\right)$ would be a flat section of $\hat{\Omega}$ in $\partial \Omega$, a contradiction.

Remark. Not all locally trivial holomorphic disc bundles $\Omega \rightarrow X$ over a compact Kähler manifold $X$ allow harmonic sections as can be seen from the following example:

We take as $X$ the elliptic curve $X=C /<1, \sqrt{-1}>$ and define $\Omega$ as upper half-plane bundle over $X$ given by the transformations $t \longmapsto t$ and $t \longmapsto t+1$.

Then, for any $C^{\infty}$-section $s: X \rightarrow \Omega$ and any $\alpha>0$ also $s+\sqrt{-1} \alpha=: s_{\alpha}$ is a well-defind section. It is easy to see that, however, $\chi\left(s_{\alpha}\right) \leqq \chi(s)$. 


\section{\$4. The Case of Flat Sections in the Associated $P^{1}$-Bundle}

The proof of theorem will be complete after we show

Proposition 3. Let $\Omega \stackrel{\pi}{\longrightarrow} X$ be as in Thm. 1. Suppose that the associated $\boldsymbol{P}^{1}$-bundle $\hat{\boldsymbol{Q}} \stackrel{*}{\longrightarrow} X$ has a flat section in $\partial \Omega$. Then $\Omega$ is weakly 1-complete.

The following lemma, which we will prove first, will make it possible to construct the desired exhaustion function on $\Omega$ almost as in the case of domains in $\boldsymbol{C}^{n}$.

Lemma 5. Let $s: X \rightarrow \hat{\Omega}$ be a flat section. Then the complex manifold $\tilde{\Omega}:=\hat{\Omega} \backslash s(X)$ has the structure of a locally trivial holomorphic $\boldsymbol{C}$-bundle over $X$ with group $G=\{z \longmapsto a z+b:|a|=1, a, b \in \boldsymbol{C}\}$.

Proof. Since $s$ is flat, it is clear that $\tilde{\Omega} \stackrel{\tilde{\pi}}{\longrightarrow} X, \tilde{\pi}=\hat{\pi} / \tilde{\Omega}$, is a locally trivial holomorphic $\boldsymbol{C}$-bundle with a trivializing covering $\left\{U_{i}\right\}$ of $X$ such that the transition function $U_{i} \cap U_{j}$ are locally constant. Since $s(X) \subset \partial \Omega$, which is a $C^{\omega}$-smooth real hypersurface in $\hat{\Omega}$, the normal bundle $N_{\infty}$ of $s(X)$ in $\hat{\Omega}$ is topologically trivial. Notice that we can associate a holomorphic line bundle $L \rightarrow X$ to $\tilde{\Omega} \stackrel{\tilde{\pi}}{\longrightarrow} X$ by ignoring the translation part in the transition function of $\tilde{\Omega} \rightarrow X$. A small calculation shows that $L$ is analytically dual to $N_{\infty}$, such that $L \rightarrow X$ is also topologically trivial. Since $X$ is Kählerian, it follows from Hodge theory, that the group of $L \rightarrow X$ can be reduced to rotations (details can, for instance, be found in [2]). Therefore, the group of $\tilde{\Omega} \rightarrow X$ can be reduced to $G$.

An easy consequence of Lemma 5 is:

Lemma 6. The manifold $\tilde{\Omega}$ is weakly 1-complete.

Proof. We choose a trivializing cover $\left\{U_{i}\right\}$ of $\tilde{\Omega} \rightarrow X$ with transition functions

$$
t_{i}=a_{i j} t_{j}+b_{i j}, \quad\left|a_{i j}\right|=1
$$

(where $t_{i}$ is the fiber coordinate over $U_{i}$ ).

Using again the Kähler structure of $X$ and Hodge theory, one can 
find pluriharmonic functions $c_{i}: U_{i} \rightarrow C$ such that

$$
b_{i j}=c_{i}-a_{i j} c_{j}
$$

on $U_{i} \cap U_{j}$ (for details see [2]).

Therefore, the function

$$
\phi:=\left|t_{i}-c_{i}\right|^{2}
$$

is a well-defined plurisubharmonic exhaustion function on $\tilde{\Omega}$.

Now we can finish the

Proof of Proposition 3. Notice that the group $G$ consists of isometries for the euclidean metric on $\boldsymbol{C}$.

Therefore, a metric $d s_{p}^{2}$ is induced on each fiber $\tilde{\pi}^{-1}(p), p \in X$. We define for $q \in \Omega \subset \tilde{\Omega}$

$$
\delta(q):=\operatorname{dist}_{\pi(q)}(q, \partial \Omega)
$$

where $\operatorname{dist}_{\pi(q)}$ is the distance in $\tilde{\pi}^{-1} \pi(q)$ with respect to $d s_{\pi(q)}^{2}$.

According to Oka, [11], the $C^{\infty}$ function

$$
\phi_{0}(q):=-\log \delta(q)
$$

is then plurisubharmonic on $\Omega$. Therefore, the function

$$
\varphi(q):=\phi_{0}(q)+\phi(q)
$$

is the desired plurisubharmonic exhaustion function on $\Omega$, because near $\infty$ the function $\phi$ grows in local coordinates like the square of the distance from $t=0$.

Remark. This argument also shows, that any locally Stein domain $D \subset \tilde{\Omega}$ has a continuous plurisubharmonic exhaustion function.

\section{§5. Some Supplements}

Using the main result of [10] we can extend Theorem 1 to the following

Theorem 3. Let $X$ be a compact complex manifold which is bimeromorphically equivalent to a compact Kähler manifold. Then any locally trivial holomorphic disc bundle $\Omega \stackrel{\pi}{\longrightarrow} X$ is weakly 1-complete. 
We give a brief sketch of the

Proof. There is a compact Kähler manifold $X^{\prime}$ with a proper holomorphic modification map

$$
f: X^{\prime} \longrightarrow X
$$

The critical set of $f$ has codimension 2 in $X$. We lift $\Omega \stackrel{\pi}{\longrightarrow} X$ and obtain

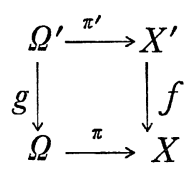

$\hat{\Omega}^{\prime}$ resp. $\hat{\Omega}$ are again the associated $\boldsymbol{P}^{1}$-bundles. Notice that one has

a) $\hat{\Omega} \stackrel{*}{\longrightarrow} X$ has a flat section in $\partial \Omega$ if and only if $\hat{\Omega}^{\prime} \stackrel{\star^{\prime}}{\longrightarrow} X^{\prime}$ has a flat section in $\partial \Omega^{\prime}$ (trivial).

b) $\Omega \stackrel{\pi}{\longrightarrow} X$ has a pluriharmonic section (in the sense of Def. 1) if and only if $\Omega \stackrel{\pi^{\prime}}{\longrightarrow} X^{\prime}$ has a pluriharmonic section. This is a consequence of the theorem in [10].

The rest of the proof is left to the reader.

In [10] the proof of the Proposition 2 was promised to be given in this paper since it is another application of our key lemma. We want to show the following extendibility result for harmonic maps:

Proposition 4. Let $h: \Delta \backslash\{0\} \rightarrow\left(\Delta, d s_{\Delta}^{2}\right)$ be a harmonic map and suppose that $h(\Delta \backslash\{0\})$ is contained in a compact set $K \subset \Delta$. Then $h$ has a harmonic extension $\hat{h}: \Delta \rightarrow \Delta$.

Proof. We use all notations of $\S 2$. It is well-known that the (potential) energy density of $h$ can be written as

$$
\chi(h)=|\partial h|^{2}+|\bar{\partial} h|^{2}
$$

where lengths are measured with respect to $d s_{\Delta}^{2}$. For any $z \in \Delta \backslash\{0\}$ we choose $T_{z} \in$ Aut $\Delta$ with $T_{z}(h(z))=0$. Then we get by applying (5a) to the harmonic map $T_{z} \circ h$ the following inequality

$$
l_{\varphi}(z, w) \geqq \frac{1}{2} \frac{|w|^{2}}{1-|w|^{2}} \chi\left(T_{z} \circ h\right)(z)=\frac{1}{2} \frac{|w|^{2}}{1-|w|^{2}} \chi(h)(z) \text {. }
$$


We choose a disc $\Delta_{0} \Subset \Delta$ with $K \subset \Delta_{0}$ and integrate (18) in $w$ over $\Delta_{0}$ :

$$
\int_{\Delta 0} l_{\varphi}(z, w) d v_{w} \geqq c \chi(h)(z)
$$

with $c>0$. Another integration over $\Delta^{\prime}(:=\Delta \backslash\{0\})$ yields

$$
\int_{\Delta^{\prime}} \chi(h)(z) d v_{z} \leqq C \int_{\Delta^{\prime} \times \Delta_{0}} l_{\varphi}(z, w) d v_{z} d v_{w}
$$

Since $\varphi$ is bounded and plurisubharmonic on $\Delta^{\prime} \times \Delta_{0}$ it extends to a bounded plurisubharmonic function on $\Delta \times \Delta_{0}$. Therefore, acoording to Bedford-Taylor [1] the right side of (18) finite, which shows that $h$ has finite energy on $\Delta^{\prime}$. Hence $h$ extends to a harmonic map from $\Delta$ to $\Delta$ according to a result of Sacks and Uhlenbeck [12].

\section{References}

[1] Bedford, E., Taylor, B. A., The Dirichlet problem for the complex Monge-Ampère equation, Invent. Math., 37 (1976), 1-44.

[2] Diederich, K., Ohsawa, T., A Levi problem on two-dimensional complex manifolds. Math. Ann., 261 (1982), 255-261.

[3] Diederich, K., Fornaess, J.E., A smooth pseudoconvex domain without pseudoconvex exhaustion, Manuscripta math., 39 (1982), 119-123.

[4] Eells, J., Sampson, J.H., Harmonic mappings of Riemannian manifolds, Amer. J. Math., 86 (1964), 109-160.

[5] Grauert, H., On Levi's problem and the embedding of real-analytic manifolds, Ann. Math., 68 (1958), 460-472.

[6] .., Bemerkenswerte pseudokonvexe Mannigfaltigkeiten, Math. Zeitschrift, 81 (1963), 377-391.

[7] Hamilton, R. S., Harmonic maps of manifolds with boundary, LN in Mathematics, vol. 471. Berlin-Heidelberg-New York 1975.

[8] Narasimhan, R., The Levi problem in the theory of functions of several complex variables, ICM Stockholm 1962.

[9] Ohsawa, T., Cohomology vanishing theorems on weakly 1-complete manifolds, Publ. RIMS Kyoto, 19 (1983), 1181-1201.

[10] . On the domain of existence for P-pluriharmonic functions, Publ. RIMS Kyoto, 20 (1984), 1021-1024.

[11] Oka, K., Domains pseudoconvexes, Tôhoku Math. J., 49 (1942), 15-52.

[12] Sacks, J., Uhlenbeck, K., The existence of minimal immersions of 2-spheres, Ann. Math., 113 (1981), 1-24.

[13] Siu, Y.T., The complex analyticity of harmonic maps and the strong rigidity of compact Kähler manifolds, Ann. Math., 112 (1980), 73-111. 
